

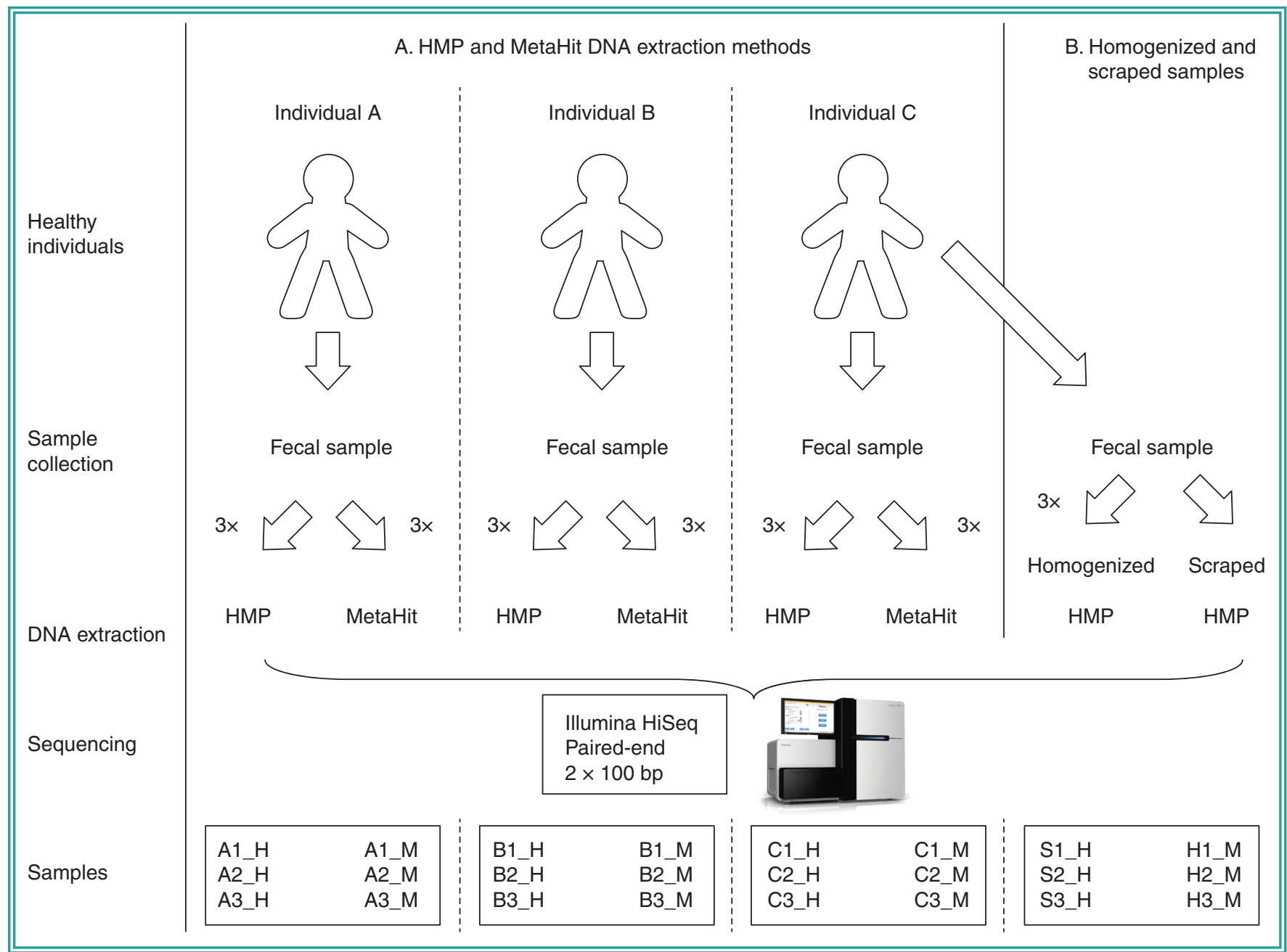

Schematic representation of the study design. (A) Comparison of the HMP and MetaHIT DNA extraction methods. (B) Comparison of homogenized and scraped samples. Reproduced from [1].

- There are numerous opportunities for experimental biases to profoundly alter the results of a study. In addition to these factors, research groups will often alter experimental processes depending on their specific study. Therefore, recognition and documentation of these factors can help towards controlling the variability between labs.

One example of interest that highlighted the severity of this issue focused on the DNA extraction methods used by two major collaborative efforts: The European MetaHIT and the American Human Microbiome Project (HMP) [1]. Interestingly, depending on the method, significant differences in the distribution of bacterial taxa were observed. For example, DNA from bacteria within the Bacteroidetes phylum was most enriched by the HMP protocol.

In another study, Costea et al. tested 21 representative DNA extraction protocols on the same fecal samples. Variations resulting from different extraction methods were then compared, with differences attributed to library preparation and sample storage. The researchers demonstrated that DNA extraction had the largest effect on the outcome of metagenomic analysis [2].

Studies that highlight these technical variations accelerate the push for standardized methods - in this case, a DNA extraction method for human fecal samples.
Kemp explained: "The lack of congruency between methods and the chronic absence of workflow controls and standards has led to protocols that are not optimized for microbiome measurements that demand new levels of rigor. Measurement accuracy and reproducibility is a serious concern for the future health of the microbiome field and, unfortunately, many are unaware of the challenges."

Kemp continued to describe what is being done to tackle this worrying issue: "Fortunately, the US National Institute of Standards and Technology (MD, USA) has been hosting workshops on standards for microbiome measurements annually to draw awareness to the problems within the field."

In an effort to improve microbiome measurement accuracy and reproducibility across the field, companies such as Zymo Research are driving initiatives to encourage the use of and increase accessibility of well-defined microbial standards.

"We are urging researchers to question and validate their methodology using mock-microbial community standards. Currently, Zymo Research is providing thousands of free microbiome standards and controls to researchers through the Microbiomics Standards \& Controls Initiative. Using microbiome standards as a ground truth, Zymo Research has built an entire workflow of highly accurate 
tools and kits for collection, extraction and library preparation for measuring a microbiome," explained Kemp.

While the goal is simple - to achieve accurate, reproducible measurements in this exciting field - the road to success might be longer than we think. It will take a combined effort between the publications, the publishers, the funders, reviewers and the institutions. "With this effort, the field will coalesce on basic standards of best practice to ensure quality measurements, such as the use of microbiome community standards as routinely as one would perform positive and negative controls," Kemp continued.

"Transparent and open sharing of quality data and materials is a cornerstone of reproducible science. Different scientific fields have different sharing cultures," commented Joanne Kamens, Executive Director of Addgene (MA, USA).

"We're always happy to see a field that is growing engage in dialog about best practices towards openness, sharing and reproducibility. We'd be eager to highlight a microbiome research collection of plasmids, should members of that community step up to help us create and curate it."

\section{SETTING THE STANDARD}

So what can we do to begin to rectify and solve some of these reproducibility issues?

Cell lines were also a subject of the ATCC survey. The problems with cell line misidentification have been known for a long time, contributing to the growing concerns about irreproducible experiments and false conclusions. The current call for action is focused on improving the verification of cell lines.

Mark Capriani, Senior Director of Marketing at ATCC, commented: "It's starting with credible materials that allows you to do incredible things. If you don't know what's in your materials, you could squander a lot of time and a lot of money trying on science that may have been compromised for a variety of reasons. Without authenticated materials, there's a variety of challenges to ensure the science is reproducible."

Interestingly, the ATCC survey also revealed an insight into the proportion of researchers who might place too much trust in their peers when borrowing cell lines: nine out of ten scientists reported borrowing cell lines from their colleagues and only $29 \%$ of them will reauthenticate before use.

Capriani also highlighted the importance of reauthenticating borrowed cell lines: "In our experience, most of the scientists who borrowed cell lines didn't seem to blame the irreproducibility of their research on technique or the protocols. There is a common misconception that you can trust cell lines from scientific colleagues without needing to reauthenticate, but it is actually distinctly possible that it is contributing to the issue."

The key to tackling the crisis lies in making authentication easy for researchers. Again, it is important to note that it is both publishers and researchers alike who need to start seeing standards as an asset rather than a chore.

\section{STEPS IN THE RIGHT DIRECTION}

As mentioned previously, the pressure to publish, fueled by the academic incentive system, encourages rapid submission of research findings to the detriment of careful replication. Limited

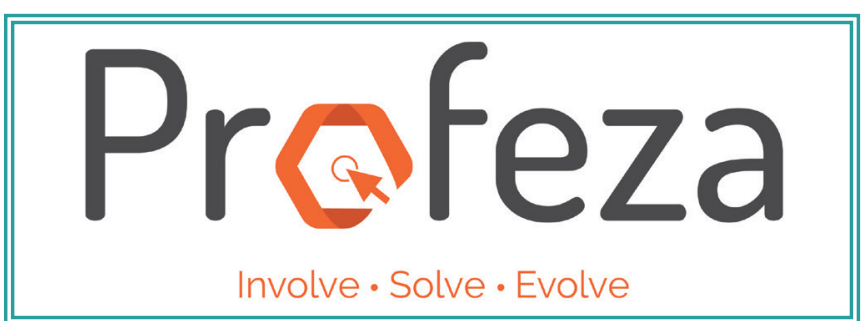

Figure 2. Profeza: a product that provides a set of workflow software tools to help create more easily reproducible protocols [4].

platforms for publishing negative data is also a problem. This is where both funding agencies and publishers can make a positive impact.

Efforts to tackle the reproducibility problem are not restricted to researchers; funding agencies are also playing their part. The $\mathrm{NIH}$ (MD, USA) is developing a training module on enhancing reproducibility and transparency of research findings, with a particular focus on good experimental design [3].

Improving data availability and protocol sharing are just two steps that can be taken by publishers to help tackle the reproducibility crisis, the latter being a recent step that BioTechniques is taking to ensure that all of the information needed to replicate a method is presented in every article.

BioTechniques has recently partnered with Profeza [4], a product that provides a set of workflow software tools to help create more easily reproducible protocols. It makes the improvement of research outputs a continuous process rather than a one-time event, allowing other researchers to follow the protocol step-by-step and provide feedback on certain aspects that either worked well or could do with improvement. The authors of the protocol are then able to constantly edit their protocol, based on their own research or feedback from others. We encourage all of our authors to submit a protocol alongside their manuscript that can then be published on Profeza [5].

Additionally, we are actively working together with members of our editorial board to create a reproducibility advisory group, highlighting the most important issues in reproducibility and what can be done from a publisher's perspective to develop clear guidelines for authors and to emphasize the importance of depositing and sharing reagents, and encouraging cell-line authentication prior to publication.

Despite the abovementioned steps, there is still a lot more work to be done and improving the reproducibility of our research outputs will remain a priority for BioTechniques.

\section{Written by Joseph Martin}

\section{REFERENCES}

1. Wesolowska-Andersen A, Bahl MI, Carvalho V et al. Choice of bacterial DNA extraction method from fecal material influences community structure as evaluated by metagenomic analysis. Microbiome 2(1), 19 (2014)

2. Costea PI, Zeller G, Sunagawa $\mathrm{S}$ et al. Towards standards for human fecal sample processing in metagenomic studies. Nat. Biotechnol. 35(11), 1069-1076 (2017).

3. Collins FS, Tabak LA. Policy: NIH plans to enhance reproducibility. Nature 505(7485), 612-613 (2014)

4. Profeza. www.profeza.com

5. Reuse Recipe Document for. TEG-seq: an ion torrent-adapted NGS workflow for in cellulo mapping of CRISPR specificity. Profeza. doi:10.23942/biotechniques.1544529037000 (2019) 